\title{
Non-invasive monitoring of spreading depression
}

Zoya J.R. Bastany ${ }^{1}$, Shahbaz Askari ${ }^{1}$, Guy Dumont ${ }^{1,2}$, Erwin-Josef Speckmann ${ }^{3}$, Ali Gorji ${ }^{3-7}$

${ }^{1}$ The Electrical and Computer Engineering Department, University of British Columbia, Vancouver, Canada

${ }^{2}$ Child and Family Research Institute, University of British Columbia, Vancouver, Canada

${ }^{3}$ Epilepsy Research Center, Westfälische Wilhelms-Universität Münster, Germany

${ }^{4}$ Shefa Neuroscience Research Center, Khatam Alanbia Hospital, Tehran, Iran

${ }^{5}$ Mashhad Neuroscience Department, MUMS, Mashhad, Iran

${ }^{6}$ Klinik und Poliklinik für Neurochirurgie, ${ }^{7}$ Klinik und Poliklinik für Neurologie, Westfälische

Wilhelms-Universität Münster, Germany

\section{Corresponding Authors:}

Prof. Dr. med. Ali Gorji, M.D.

Epilepsy Research Center,

Westfälische Wilhelms-Universität Münster,

Münster, Germany

E-mail: gorjial@uni-muenster.de

Tel: +49-251-8355564 


\begin{abstract}
Spreading depression (SD), a slow propagating depolarization wave, plays an important role in pathophysiology of different neurological disorders. Yet, research into SD-related disorders has been hampered by the lack of non-invasive recording techniques of SD. Here we compared the manifestations of SD in continuous non-invasive electroencephalogram (EEG) recordings to invasive electrocorticographic (ECoG) recordings in order to obtain further insights into generator structures and electrogenic mechanisms of surface recording of SD. SD was induced by $\mathrm{KCl}$ application and simultaneous SD recordings were performed by scalp EEG as well as ECoG electrodes of somatosensory neocortex of rats using a novel homemade EEG amplifier, $\mathrm{AgCl}$ recording electrodes, and high chloride conductive gel. Different methods were used to analyze the data; including the spectrogram, bi-spectrogram, pattern distribution, relative spectrum power, and multivariable Gaussian fit analysis. The negative DC shifts recorded by scalp electrodes exhibited a high homogeneity to those recorded by ECoG electrodes. Furthermore, this novel method of recording and analysis was able to separate SD recorded by scalp electrodes from non-neuronal DC shifts induced by other potential generators, such as the skin, muscles, arteries, dura, etc. These data suggest a novel application for continuous non-invasive monitoring of DC potential changes, such as SD. Non-invasive monitoring of SD would allow early intervention and improve outcome in SDrelated neurological disorders.
\end{abstract}

Key words: Brain waves, Migraine, Stroke, Slow wave, Epilepsy, Ultraslow wave 


\section{Introduction}

Voltage fluctuations generated by ionic currents within the brain are comprised of sustained displacements and slow fluctuations as well as faster waves up to at least $100 \mathrm{~Hz}$ superimposed on deviations of the baseline. These bioelectrical activities are comprised of conventional electroencephalogram (EEG) waves and of slow potentials. Recording of slow components of brain activity are often distorted by artifacts generated by the body (e.g. movements of eyes and head, muscle contractions). This has led to the introduction of voltage amplifiers with high- and low-filters that eliminate components below and above given frequencies and thus stabilizes the baseline. The EEG band pass is considered between 0.2-90 $\mathrm{Hz}$, with the frequencies out of this range being eliminated. The high-pass filter (usually set at 0.5-1 Hz) filters out slow waves or artefacts, and a low-pass filter (usually set at 35-70 Hz; Niedermeyer and da Silva, 2004) eliminates high-frequency fluctuations and power line interferences (Caspers et al., 1980; Caspers, 1993).

A functional unit consisting of neurons, glial cells, and blood-brain barrier, acts as a compound generator of DC potentials. Investigation of the slow components of the cortical DC potentials, although often neglected, is a valuable source for understanding of higher brain functions as well as the pathophysiology of different neurological disorders. These slow waves reflect changes in the level of excitation and excitability of the cortical and subcortical structures. DC potentials are correlated with voluntary muscle movement (Cui and Deecke, 1999), state of high alertness (Bachmann, 1984), task performance (Trimmel et al., 2001), motivational state (Hallschmid et al., 2001), behavior (Haider et al., 1981), and several other variables in the healthy human brain.

DC potential displacements have been observed in several pathologic conditions affecting the brain. Epileptiform field potentials, either focal or generalized discharges, are always associated with characteristic deviations of the DC potential in different animal models of 
epilepsy (Walden and Speckmann, 1988) as well as in the human epileptic brain (Rodin et al., 2008). A reduction in the oxygenation of the brain evokes negative DC displacements in both in vivo and in vitro animal models (Speckmann et al., 2011). The same negative DC deflections were reported in the human brain in vitro (Schmidinger et al., 1998) and in patients suffering from hypoxic brain injuries (Dreier et al., 2009).

Spreading depression (SD), a depolarization wave that propagates at $3-5 \mathrm{~mm} / \mathrm{min}$ in the neuronal tissues, has been shown to play a crucial role in the aura phase of migraine, cerebrovascular diseases, and epilepsy (Gorji, 2001). It has been shown that SD is a correlate of the aura phase in migraine (Hadjikhani et al., 2001), a predictor of poor functional outcome in cerebrovascular diseases and traumatic brain injury (Hartings et al., 2011) as well as a risk factor for late post-hemorrhagic seizures (Dreier et al., 2012). Electrocorticography (ECoG) as well as deep electrodes have been used for most of SD recording in animal and human brain. Non-invasive recording of DC potentials and SD by magnetoencephalography have been reported (Bowyer et al., 2012). Using conventional EEG, investigations on generator structures and mechanisms of DC potentials meet with several difficulties. Non-invasive recording of DC field potentials raises the issue whether and to what extent DC field potentials recorded from the scalp represent the changes in cortical and subcortical DC potentials (Tomita-Gotoh and Hayashida, 1996). Some studies indicate that negative DC potentials recorded non-invasively at the surface of the scalp during SD do not always reflect the activation state of the brain (Lehmenkuehler et al., 1999). A main problem is that sustained or slowly changing DC potentials picked up from the scalp can originate from a variety of generators, such as the skin, muscles, arteries and dura (Caspers et al., 1984). Using a novel method, the aim of this study was to compare the SD waves recorded from the scalp and ECoG in order to provide further insights into generator structures and electrogenic mechanisms of these negative DC potentials. 


\section{Materials and methods}

All experiments were conducted in accordance to the guiding principles for the care and use of animals in the University of Münster, Germany (50.0835.2.0/A-18/2006), by the Animal Care Committee of University of British Columbia, and Shefa Neuroscience Research Center, Tehran, Iran. Twenty adult Wistar rats (210-400 g) were housed individually under controlled environmental conditions (12-h light/dark cycle) with food and water available ad libitum. The animals were anesthetized with intraperitoneal injection of chloral hydrate $(350 \mathrm{mg} / \mathrm{kg}$ Sigma-Aldrich) and the head of each rat is placed in a stereotaxic instrument (Stoelting Instruments, USA). This dose of chloral hydrate provides sufficient deep anesthesia for surgical procedures (Field et al., 1993). Soft tissues of the skull at the incision and at contact points were anesthetized using local application of $0.5 \%$ lidocaine hydrochloride. Additional supplemental doses of lidocaine and chloral hydrate were applied when necessary (Koulchitsky et al., 2012).

Stainless steel guide cannula and three $\mathrm{Ag} / \mathrm{AgCl}$ recording electrodes (2-3 mm apart) were implanted above the somatosensory neocortex. Three electrodes were positioned next to the cannula, which were designated ECoG, scalp (EEG)1, and scalp (EEG)2. Craniotomy exposed the surface of the neocortex for insertion of cannula (used for application of $\mathrm{KCl}$ ) and a further 2-3 mm posteriorly, for an $\mathrm{Ag}-\mathrm{AgCl}$ electrode to record surface DC potentials after drilling small holes in the skull and removing the dura (ECoG electrodes; $\mathrm{Ag} / \mathrm{AgCl}$ ball on a flexible silver wire, $1 \mathrm{~mm}$ diameter). Scalp recordings electrodes ( $\mathrm{Ag} / \mathrm{AgCl}$ disc-recording electrodes, $2 \mathrm{~mm}$ diameter) were positioned posterior to ECoG electrode on the depilated skin. $\mathrm{An} \mathrm{AgCl}$ reference electrode was implanted in the nasal cavity.

\section{Recording of negative DC potentials}

\section{EEG Amplifier}


Electrodes were connected to a novel purpose build AC/DC EEG amplifier. For design of this novel amplifier several points were taken into consideration. EEG noise reduction was the main challenging part in designing the amplifier. Other challenges encountered during the design of this amplifier were: $i$ ) amplification of the large amplitude range (20 $\mu \mathrm{V}-30 \mathrm{mV}), i i)$ the amplifier saturation caused by electrode and tissue due to elimination of the high pass filter, iii) the achievement of a high Common Mode Rejection Ratio (CMRR) in low ADIF (differential gain), and $i v$ ) control of the electrode potential stability during DC recording of bioelectrical activities. Various known pathological DC potentials can be produced in the brain, such as a DC potential shift during seizure activities (amplitude up to $500 \mu \mathrm{V}$ and duration up to $16 \mathrm{~s}$ ) or $\mathrm{SD}$ (the amplitude of 2 to $30 \mathrm{mV}$ and duration of $30-120 \mathrm{~s}$ ). On the other hand, in $\mathrm{AC}$ recording mode, the frequency of interest is typically in the $0.5-70 \mathrm{~Hz}$ range with an amplitude range of $20 \mu \mathrm{V}-600 \mu \mathrm{V}$. Therefore, the AC/DC amplifier should amplify the bioelectrical signals across these boundaries in order to be capable of both AC and DC EEG data recording. In order to eliminate the common mode artifacts, a high CMRR of $>100 \mathrm{~dB}$ with low absolute values of differential gain were used.

As the potentials of the electrode-electrolyte-tissue interface do not change symmetrically, currents in the input circuit must be kept as low as possible to minimize slow drift artifacts. Moreover, the electrode impedance is higher in the slow frequencies; therefore, the amplifier input impedance should be higher than $1 \mathrm{G} \Omega$ to minimize this effect. The control and measurement of electrode potential stability are other important steps toward obtaining stable DC recordings. In this novel AC/DC EEG amplifier, CMRR >110db with differential gains of 20 for the preamplifier and 10 for the amplifier, and sensitivity of $20 \mu \mathrm{V}-40 \mathrm{mV}$ were considered. The input impedance is $>10 \mathrm{G} \Omega$, bias currents of $10 \mathrm{pA}$ maximum, the lowfrequency cutoff is $<0.015 \mathrm{~Hz}$, and a driven right leg circuit (DRL) was used as a connection between the signal source and the common of amplifier to reduce the common mode voltage. 
A software and an analog-to-digital (ATD) converter (NRSIGN INC, BC, Canada) with the ability of programming the high pass filter from $0-3 \mathrm{HZ}$, page time scaling up to $1 \mathrm{~mm} / \mathrm{s}$, sample rate of 500-2 k sample/s, ATD resolution of 24 bits, and 50/ $60 \mathrm{~Hz}$ notch filter were used for data recording.

\section{Electrode specification}

Sintered $2 \mathrm{~mm}$ diameter $\mathrm{AgCl}$ disc recording electrodes for scalp (EEG) recordings and $\mathrm{AgCl}$ wire for ECoG recording were used. Changes in the half-cell potential are a main source of DC voltage drifts, which can ruin the DC potential recordings. $\mathrm{AgCl}$ electrodes are nonpolarizable with a low half-cell potential. $\mathrm{AgCl}$ electrodes have more stable resistance in long-term recordings with lower drift rates and therefore are able to minimize noise, particularly during recording of slow frequency potentials (Tallgren et al., 2005). When a DC potential is recorded by a scalp electrode placed in an electrolyte solution, the electrical double layers are disturbed and a current flows. The magnitude of this current as well as the ultimate electrical impedance of electrodes is dependent on ion transport at metal-electrolyte junction (Kamp et al., 2004). To obtain an appropriate resistance with a reasonable stability, an electrolyte with high concentration of chloride is suggested (Almasi and Schmitt, 1970). Ten 20 gel (Weaver and Company, Colorado, USA) was used as electrode gel in this study as it has the highest concentration of $\mathrm{Cl}^{-}$with lowest drift rate among commercially available gels (Tallgren et al., 2005).

\section{Induction of SD}

SD was induced by application of $3 \mathrm{M} \mathrm{KCl}$. A stylet was placed into the guide cannula to allow it to maintain potency. The stylet was withdrawn from the guide cannula, and a 27gauge injection needle inserted. The injection needle was attached to a $10 \mu$ Hamilton syringe by a polyethylene tube (Harvard Apparatus, Inc.). $\mathrm{KCl}$ solutions were injected in a total 
volume of $10 \mu \mathrm{l}$. The injection needle was retained in the guide cannula for an additional $60 \mathrm{~s}$ after injection to facilitate diffusion of $\mathrm{KCl}$. Negative $\mathrm{DC}$ events were evaluated with respect to their amplitude, duration, and velocity rates. The SD amplitude is considered as peak-topeak voltage of the signal. The criteria for identifying DC shifts as SD were high amplitude (up to $30 \mathrm{mV}$ ) as well as long duration (up to 120s) of negative DC fluctuation, slow propagation of DC waves between the electrodes, and inhibition of the normal pattern of EEG and ECoG activities (Gorji et al., 2001). To determine DC shifts due to SD from normal EEG fluctuations, mean and standard deviation of the DC potential during normal EEG was calculated during 5 seconds before $\mathrm{KCl}$ injection. The recorded data were exported to MATLAB (The Mathworks, Inc.) software and the mean and standard deviation were calculated. In Figure 1, the solid green line is the mean $\mu$ of the first 5 seconds of the signal and the dashed lines are the mean and standard deviation $(\mu+\sigma$ and $\mu-\sigma)$ of the EEG signal amplitude. We define the start of SD signal as the moment the EEG signal crosses one of dashed lines and starts to assume the shape of the standard DC potential morphology (the first or the second component). The end of the DC potential signal is marked when the negative low amplitude component (the third component) of the DC signal returns between the dashed lines (Fig. 1). Each electrode has 3 coordinates defined as horizontal, vertical and depth; and the distance between the electrodes was measured based on the coordinates of each electrodes. Low threshold crossing algorithms was selected to evaluate the traces as the impact of lower/higher threshold was rather small in our study. According to the defined method in the power spectral analysis (see below), the time differences between the maximum energy of signals were considered as the time delay between them. From the distance between the electrodes and the time delay between the energy of the signals, the propagation velocity was measured. Our calculation is based on the assumption that the SD spreads in parallel with the orientation of the electrodes. 


\section{Spectrogram Analysis}

Spectral analysis is one of the commonly used methods to explore a signal's components in the frequency domain. The spectrogram is an acceptable visual representation of the power density of the non-stationary signals in the frequency domain, and can present the frequency variations of negative DC potentials recorded from multi-electrodes. The spectrogram has three axes, the horizontal axis represents time, while the vertical axis represents different frequencies, and the color intensity shows the amplitude of the signal power at the corresponding time and frequency. To calculate the spectrogram of the signals, a Kaiser window was used and the windowed signal was represented in Eq. 1.

$$
x_{w}(\tau)=x(t) * W(t-\tau)
$$

The fourier transform of the windowed signal was calculated using Eq. 2, and the spectrogram of the signal was achieved by applying Eq. 3 .

$$
\begin{aligned}
& X_{w}(f, \tau)=\int_{-\infty}^{+\infty} x_{w}(\tau) e^{-j 2 \pi f t} d t \\
& S_{w}(f, \tau)=E_{f}\left[\left|X_{w}(f, \tau)\right|^{2}\right]
\end{aligned}
$$

The simultaneous recording of the SD was imported to MATLAB. The data were sampled at $500 \mathrm{~Hz}$ and down sampled between $10-50 \mathrm{~Hz}$ before analysis of the spectrogram. To eliminate the power line and unwanted noise a $50 \mathrm{HZ}$ notch filter and a low-pass filter with cut-off frequency at $35 \mathrm{~Hz}$ were applied to the signal, while no high-pass filtering was applied. In the spectrogram analysis, the Kaiser window $(\mathrm{N}$ (the width) $=100, \beta$ (the shape) =3) was used.

\section{Bi-spectral analysis}

Spectral analysis loses the phase information, as it is a second order statistical analysis from the autocorrelation. The bi-spectrum is an informative higher order statistical analysis and 
preserves the Fourier phase information. Therefore, this method is capable of extracting the frequency change of SD waves during different phases of SD waves (before initiation, during neuronal excitation, depolarization, depression, and recovery phases). The analysis aids to verify that the SD signals recorded from multi- electrodes are initiated from a common source and have a same morphology. Eq.4 shows the calculation of the bi-spectrum using Fourier transforms evaluated at $\mathrm{f}_{1}$ and $\mathrm{f}_{2}$ (Wong al., 1999).

$$
B\left(f_{1}, f_{2}\right)=\sum_{\tau_{1}=-\infty}^{\tau_{1}=+\infty} \sum_{\tau_{2}=-\infty}^{\tau_{2}=+\infty} C^{3}\left(\tau_{1}, \tau_{2}\right) e^{\left.\left.-j\left(\tau_{1} \mathrm{f}_{1}\right)+\tau_{2} \mathrm{f}_{2}\right)\right)}
$$

where $C^{3}\left(\tau_{1}, \tau_{2}\right)$ is the third order cumulant of a stationary signal, for real-value and stationary signal $x$, the cumulant can be calculated from the Eq.5.

$$
C^{3}\left(\tau_{1}, \tau_{2}\right)=E\left\{x(t) x\left(t+\tau_{1}\right) x\left(t+\tau_{2}\right)\right\} \quad(E q .5)
$$

$\mathrm{E}\{\}$ is the statistical expectation. As can be deduced from the equation when the signal is stationary, the cumulant is dependent on two time lags $\left(\tau_{1}, \tau_{2}\right)$. As two shifted signals are involved in the formula, the bi-spectrum provides a decomposition of the third moment. The cumulant and correspondingly bi-spectrum are 2D matrix and have symmetrical properties.

In bi-spectrum calculation of the non-stationary signals, a window function that meets the symmetry properties of the cumulant is used, and the 2D window was constructed from the one-dimensional window with Eq.6 (Abeyratne al., 1999).

$$
w(m, n)=d(n) d(m) d(n-m)
$$

where $\mathrm{d}$ is the one- dimensional window function, $\mathrm{n}$ and $\mathrm{m}$ are the number of samples along the vertical and horizontal axis; a Parzen window with 40 samples was used in this study.

\section{Correlation and Multivariate Gaussian Fit Analysis}


The correlation coefficient between the amplitude and the duration of the traces recorded from ECoG and scalp were calculated. In addition, a multivariate Gaussian was fitted to analyze the consistency and distribution of the data recorded by multi-electrodes. The multivariate Gaussian distributions were fitted to the farthest, the nearest, and the middle of the data points.

\section{Pattern related event distribution of DC potentials}

The signal amplitude (ECoG and EEG) was normalized, and then shifted in the time domain so that their maximum amplitudes were at one point. Then the signals were divided between $2 \%$ to $10 \%$ of the SD duration (depending on their resolution) and two methods were used for comparison of the amplitudes. The first method was to calculate amplitude differences of normalized signals and the amplitude comparison was given by the ratio between the integral of those differences and the integral of the first signal (Eq.7-9)

$$
\begin{aligned}
& \mathrm{S}_{\text {Diff }}^{\mathrm{i}}=\int_{\mathrm{t}_{\mathrm{i}}}^{\mathrm{t}_{\mathrm{i}+1}} \operatorname{Sig}_{\mathrm{n}}^{\mathrm{ECoG}}-\operatorname{Sig}_{\mathrm{n}}^{\text {Scalp }} \\
& \mathrm{S}_{\mathrm{ECoG}}=\int_{0}^{\mathrm{T}} \operatorname{Sig}_{\mathrm{n}}^{\mathrm{ECoG}} \\
& R_{i}=\frac{S_{D i f f}^{i}}{S_{E C o G}}
\end{aligned}
$$

where $t_{i}$ stands for $\mathrm{i}^{\text {th }}$ percentage of the signals.

The second method was calculating the ratio of the local maximum and the global maximum of the signal in each time epoch (Eq.10). The comparison of two signals is given by subtraction of their ratio:

$$
\begin{aligned}
& R_{i}=\frac{M_{E C o G}^{i}}{M_{E C o G}^{g l o b a l}} \\
& M_{E C o G}^{\text {global }}=\max _{\mathrm{t} \in(0, \mathrm{~T})}\left(\operatorname{Sig}_{n}^{E C o G}\right) \\
& \mathrm{M}_{\mathrm{ECoG}}^{\mathrm{i}}=\max _{\mathrm{t} \in\left(\mathrm{t}_{\mathrm{i}}, \mathrm{t}_{\mathrm{i}+1}\right)}\left(\operatorname{Sig}_{\mathrm{n}}^{\mathrm{ECoG}}\right)
\end{aligned}
$$




\section{Results}

Recording of bioelectrical activities

After $20 \pm 10 \mathrm{~s}$ of local applications of $\mathrm{KCl}$ on the dura overlying the somatosensory cortex, the negative DC deflections followed by positive waves were recorded by ECoG electrodes, (amplitude of $7.4 \pm 0.8 \mathrm{mV}$; duration of $73.8 \pm 4.9 \mathrm{~s}$ ). Then, scalp (EEG)1 electrodes recorded a negative DC potential with the amplitude of $2.2 \pm 0.2 \mathrm{mV}$ and duration of $56.6 \pm 5.3 \mathrm{~s}$. The velocity of propagation of DC potentials between ECoG and scalp (EEG)1 electrodes was 4.2 $\pm 0.5 \mathrm{~mm} / \mathrm{min}$. Finally, the negative DC deflections were recorded by scalp (EEG)2 electrodes with the amplitude of $1.4 \pm 0.1 \mathrm{mV}$ and duration of $49.6 \pm 5.9 \mathrm{~s}$. The velocity of DC potential propagation between scalp 1 and scalp 2 electrodes was $6.2 \pm 0.7 \mathrm{~mm} / \mathrm{min}$ (Fig. 2 A). The negative deflections of DC shifts were in the same direction in ECoG and scalp recordings. However, the amplitude of DC fluctuation was smaller and the duration became progressively transient in ECoG and scalp recordings (Fig. 2 A). During appearance and propagation of negative DC potentials, recording with a high-pass filter of $<0.015 \mathrm{~Hz}$ revealed reduction of bioelectrical activities that propagated consequently from ECoG recording site toward scalp2 electrode (Fig. 2 A).

\section{Spectrogram analysis}

A high energy of signal in a frequency of less than $3 \mathrm{~Hz}$ was observed after $\mathrm{KCl}$ injection in the spectrogram of signals recorded by ECoG electrodes. The spectrogram of signals recorded during initiation and propagation of SD revealed high-energy signals in a frequency between 0.05 to $0.1 \mathrm{~Hz}$. The same pattern of high-energy signals of less than $0.1 \mathrm{~Hz}$ was observed in the spectrogram of signals recorded by scalp (EEG) electrodes during propagation of SD (Fig. $2 \mathrm{~B})$. Although the energy of signals of less than $0.1 \mathrm{~Hz}$ in the spectrogram of both ECoG and scalp recordings reduced after returning of DC potential shifts to pre-SD level, however, the intensity of these signals was continued for 30 to $360 \mathrm{~s}$. The intensity of signals over $1 \mathrm{~Hz}$ 
markedly decreased during and after propagation of negative DC potentials (Fig. 2 B). The brain electrical activity tends to be recovered about 150 seconds after SD propagation in the scalp 2 electrode. The recovery was observed in both ECoG and scalp electrodes. After adjustment of the power spectrum from $35 \mathrm{~Hz}-4 \mathrm{~Hz}$ (Fig. 2 B), frequency signals between 2-4 $\mathrm{Hz}$ were observed during the SD wave, which can be ascribed to the vital activities.

\section{Bi-spectral analysis}

Bi-spectral analysis showed the changes of brain wave frequencies before, during, and after appearance of negative DC deflection. The specified time for bi-spectral analysis is selected according to SD feature (Fig. 3). The presence of lower frequencies and simultaneously disappearance of higher frequencies of bioelectrical activities of the brain at the beginning of negative DC potentials recorded by ECoG and scalp electrodes are shown in Fig. 3 B-E and Fig. 4 C-E, respectively. The green and light blue colors and some small red contours around the main central red ellipse indicate the emergence of higher frequency components before appearance of negative DC potentials. During and after SD-like wave, this red ellipse started to being more confined and the small red contours around the main counter were completely disappeared in both ECoG and scalp recordings. Meanwhile, dark and light blue contours were just limited around the center of the bi-spectrum pattern. This indicates that the high frequency components were eliminated during SD-like wave in both ECoG and scalp recordings (Fig. 3 C-E and Fig. 4 C-F). ). After 200-300 s of initiation of negative DC potentials, the higher frequency components were returned to pre-SD-like deflection state (Fig. 3 G-L and Fig. 4 G-K). This can be attributed to the recovery state after neuronal depression. The results of $\mathrm{Bi}$-spectral analysis indicated that the ECoG and EEG signals followed the same patterns during different phases of the SD event.

\section{Correlation and multivariable Gaussian fit analysis}


The correlation of the amplitude and duration of bioelectrical traces recorded by different electrodes was performed. The correlation between the amplitude and duration of negative DC potentials recorded by ECoG and scalp electrodes revealed a negative correlation of 0.88 and a positive correlation of 0.908 , respectively (Fig. 5). Furthermore, there was a positive correlation of 0.94 between the amplitude of SD-like deflections recorded by scalp 1 and scalp 2 electrodes. A positive correlation of 0.92 was observed between negative DC waves recorded by scalp 1 and scalp 2 (Fig. 5).

\section{Pattern related event distribution of DC potentials}

The recorded ECoG and scalp DC potentials were synchronized, normalized, and compared for changing of signal morphology. The homogenous distribution of negative DC shifts recorded by ECoG-EEG electrodes (Fig. 6), and for scalp (EEG) 1-scalp (EEG) 2 (Fig. 7) were analyzed. This revealed that negative DC potentials recorded by ECoG and scalp electrodes were mainly produced by a similar generator (KCl-induced SD-like wave). However, comparison of normalized and synchronized ECoG and EEG signals as well as EEG signals recorded by two scalp electrodes revealed additional potentials, which were recorded by scalp electrodes. These small negative DC potentials may be induced by other generators of DC potentials, such as arteries, muscle, dura, etc. (Fig. 6 and 7). 


\section{Discussion}

The present data show that cortical negative DC shifts induced by $\mathrm{KCl}$ injection can be measured at the intact skin overlying the neocortex. DC potentials at the cortical surface (ECoG) showed a high homogeneity to those measured on the skin (EEG). Using novel methods of recording and analysis of brain activities, negative DC potentials picked up noninvasively at the surface of the scalp reflect the alterations of neocortical activities/excitabilities during propagation of SD waves. Furthermore, these novel methods have the potential to separate neuronal DC potentials (SD) from non-neuronal DC shifts induced by skin, muscle, arteries, dura, etc. occurring during SD.

Over the past 70 years, numerous investigations have indicated that DC potentials play an important role in both physiology and pathophysiology of the brain. As non-invasive recordings of DC potentials were compromised by large noise and artefacts that cause baseline drift, most of EEG measurements were made by AC-coupled mode, typically with high-pass filter to block out undesirable noise (Barkley et al., 1991). This, however, also obscures all brain waves occurring at frequencies less than $1 \mathrm{~Hz}$, including SD. Several attempts were made for non-invasive recording of $\mathrm{SD}$, which first was described by Leao in 1944. However, it was concluded that the detection of SD with standard methods is probably impossible (van Harreveld and Stamm, 1954; Caspers et al., 1984; Barkley et al., 1991) as instability of the electrode-skin unit changes the half-cell potential and induces baseline drifts of a few millivolts that is much higher than the bioelectrical signals of the brain (Caspers et al., 1984, Chakkalkal et al., 1988, Barkley et al., 1991). Using a glass electrode placed on the cranium, negative DC potentials were picked up at the surface of the scalp or of the skull during hypoxia-induced SD (Lehmenkuehler et al., 1999). However, negative DC potentials picked-up at scalp did not reflect the SD wave of the neocortex, indicating contamination of signals by other DC-sources. 
It has been noted that surface recording of DC potentials is critically dependent on the choice of electrode and gel (Tallgren et al., 2005). The best performing surface electrodes for very low to high frequency bioelectrical potential recordings are sintered $\mathrm{AgCl}$ electrodes (Macy, 2015; Tallgren et al., 2005). Our study showed that $\mathrm{AgCl}$ scalp electrodes are suitable for recording of SD. Due to low junction potential, the flow of current through these nonpolarized electrodes and electrolyte does not develop overpotential. The $\mathrm{AgCl}$ layer at the electrode-electrolyte junction reduces the noise in the junction and creates very stable recordings of bioelectrical potentials (Kappenman and Luck, 2010). However, a sufficiently high concentration of $\mathrm{Cl}^{-}$in the electrolyte is crucial for the stability of these electrodes during recordings of potentials. The half-cell potential is dependent on the basis of the conducting electrolyte and high $\mathrm{Cl}^{-}$ionic concentration reduces the half-cell potential. Another crucial property of an electrode for recording of DC potential is its impedance. Propagation of SD is accompanied by transient impedance increases of 10 to $30 \%$ closely followed by the slow potential deflection (Bures et al., 1974). The $\mathrm{AgCl}$ layer reduces the impedance of the electrode and decreases the noise and motion artifacts; an ideal condition for recording of DC potentials (Mansfeld et al., 1988; Vanhatalo et al., 2005; Lee and Kruse, 2015).

Although an appropriate stability in DC potential recording can be achieved by using $\mathrm{AgCl}$ electrodes and high $\mathrm{Cl}^{-}$containing gels, there are other factors that have to be taken into consideration when performing SD recordings on scalp. A higher electrode-electrolyte impedance and a negative phase of about $-1^{\circ}$ have been reported in frequencies less than 0.1 HZ (Geddes, 1997; Saadi and Attari, 2013). Therefore, the input impedance was adjusted at higher than $10 \mathrm{G} \Omega$ in our novel amplifier. AC EEG sensitivity is in the interval of 20-600uv and DC EEG amplitude can reach to $30 \mathrm{mV}$, therefore in order to cross these boundaries in our AC/DC EEG, the ADIF is diminished. In order to further reduce the common mode noise in the low ADIF, we kept the CMRR> $110 \mathrm{~dB}$ at $50 \mathrm{HZ}$ by matching closely the input 
impedance and capacitance and placing them beside the inputs pins. In addition, the input bias current was adjusted to less than $10 \mathrm{pA}$ to minimize the slow drift artifacts. The low noise, low power consumption amplifier (1.3mA/ channels) as well as shielded wires were also beneficial in achieving a stable surface recording of SD.

Transient depression of bioelectrical activities, which is maintained after 2 to 5 minutes and spreading of depolarization waves at a velocity of $3-5 \mathrm{~mm} / \mathrm{min}$ across the cortical surface are accounted for the descriptive name of SD. SD consists of a surface negative fluctuation attaining 5 to $15 \mathrm{mV}$ followed by a long lasting positivity of 1 to 2 minutes (Leao, 1944; Bures et al., 1974; Somjen, 2001). DC potential shift is an indicator of the changes of neocortical excitability, with a negative shift (early component) indicating an increased neuronal excitability and a positive shift pointing to a reduced activity (Caspers, 1993, Seghatoleslam et al., 2014; Eickhoff et al., 2014; Mesgari et al., 2015). Our homemade novel EEG amplifier was able to record the SD wave with the length of 90s and more as the high pass filter was completely eliminated in pre-amplifier and the cut-off frequency was kept at 0.015 HZ for high pass filter in the amplifier. The ADIF was diminished to a total gain of 190 and therefore the sensitivity of the amplifier was between $20 \mu \mathrm{V}$ to $30 \mathrm{mV}$. Electrophysiological recordings and spectrogram analysis are clearly showed the propagation pattern of SD waves at very low velocity between ECoG and surface electrodes. A propagating high energy at frequency of less than $0.1 \mathrm{~Hz}$ accompanied by vanishing of the signals at higher frequency was observed after initiation of SD in spectrogram analysis of ECoG and surface recordings. Furthermore, bi-spectral analysis indicated the emergence of lower frequencies and simultaneously disappearance of higher frequencies shortly after induction of SD, which was subsided by complete depression of bioelectrical activities for a period of 2-3 minutes. These findings demonstrated that negative DC fluctuations recorded by surface electrodes represented the characteristic features of SD. 
Analysis of traces recorded by ECoG and EEG electrodes revealed a relative homogenous distribution of negative DC shifts and the same morphology of SD waves. However, in addition to the brain, there are other structures that generate DC potentials. The blood-brain-barrier, muscles, and skin are significant sources of sustained and slowly changing potentials (Staschen et al., 1987; Caspers, 1993; Tallgren et al., 2005). Therefore, recording of SD by scalp electrodes could be contaminated by DC potentials induced by other sources. Our data revealed the presence of non-neuronal DC potentials in EEG samples recorded by scalp electrodes. Our method has the potential to distinguish neuronal from non-neuronal DCsources occurring during SD. A temporal correlation between different DC potentials occurring simultaneously could be used to reveal whether and to what extent SD waves are superimposed on DC potentials initiate simultaneously from other sources (Knoll et al., 1974). Isolation of SD potential pattern from irregular background activity, undertaken by means of normalization and synchronization, could provide further information as to basic mechanisms as well as the source of DC potentials (Knoll and Speckmann, 1973). In our study, analysis of voltage-time-function of signals recorded by ECoG and scalp electrodes revealed an additional DC potential, which was recorded by scalp electrodes. This indicates that although the SD wave captured by scalp electrode was contaminated by an extra DC potential induced by other generators, the main signals represents the same pattern of SD propagated in the brain.

There are some possible limitations to our study. The exact source of SD initiation as well as the distance to recording electrodes was known as $\mathrm{SD}$ was induced by $\mathrm{KCl}$ application. There might a different pattern if the source of the SD waves is unknown (spontaneous SD). Intracortical DC recordings have shown that SD-related DC shifts in superficial layers of the gray matter were not always reflected the DC shifts in deeper layers (Lehmankühler and Richter, 1993). The scalp recordings might not be adequately recorded DC shifts in deeper 
cortical layer or small-amplitude DC shifts originated far from recording site. This study was performed in anesthetized rats. The pattern of inter-relation between scalp EEG and ECoG might be different in awake animal or larger mammals. This needs to be further investigated.

Invasive recordings from the human brain revealed the importance of SD occurrence in the basic mechanism, pathophysiology, and prognosis of some neurological disorders, such as brain trauma, cerebrovascular diseases, migraine, and epilepsy (Gorji, 2001, Hartings et al., 2011; Dreier et al., 2012). Recent studies demonstrated that SD (including DC-shifts) can be recognized in human scalp EEG recordings (Drenckhahn et al., 2012; Hartings et al., 2014). Using the full-band DC/AC-EEG and sintered $\mathrm{Ag} / \mathrm{AgCl}$ electrodes, DC potential changes (identifying as spreading depolarizations) were recorded electrocorticographically in patients with aneurismal subarachnoid haemorrhage and malignant hemispheric stroke (Drenckhahn et al., 2012) as well as in patients suffering from brain trauma (Hartings et al., 2014). Negative DC deflections and depressions of spontaneous activity show correlates in human scalp direct and alternating current electroencephalography. In these two promising investigations EEG expressions of DC deflections were examined in relation to the ground truth revealed in simultaneous ECoG recordings and further studies are needed to develop specific criteria to identify negative DC shifts, such as SD, based on EEG alone (Drenckhahn et al., 2012; Hartings et al., 2014).

The present non-invasive method of negative DC potential recording and data analysis could be relevant for application of non-invasive DC EEG. The technology outlined here could be applied for continuous non-invasive EEG monitoring in a multitude of clinical settings; i.e. in early detection of focal seizures which are preceded by slow DC shifts (Ikeda et al., 1996; Dreier et al., 2012) or in the setting of cerebrovascular accident for early detection of delayed brain ischemia which has been a focus in recent studies (Gollwitzer et al., 2015), and could be 
used for recording of DC potentials occurred during physiological events (Khaleghi Ghadiri et al., 2009). 


\section{Acknowledgment}

A part of this study was supported by Iran National Science Foundation (INSF). There is no conflict of interest by authors. 


\section{References}

Abeyratne U R (1999) Blind reconstruction of non-minimum- phase systems from 1-D obliqueslices of bispectrum. IEE P-Vis Image Sign 146:253-264.

Almasi JJ, Schmitt OH (1970) Systemic and random variations of ECG electrode impedance. Ann N Y Acad Sci 170:509-519.

Barkley GL, Moran JE, Takanashi Y, Tepley N (1991) Techniques for DC magnetoencephalography. J Clin Neurophysiol 8:189-99.

Bowyer SM, Shvarts V, Moran JE, Mason KM, Barkley GL, Tepley N (2012) Slow brain activity (ISA/DC) detected by MEG. J Clin Neurophysiol 29:320-6.

Bures J, Buresova O, Krivanek J (1974) The Mechanisms and Applications of Leao's Spreading Depression of Electroencephalographic Activity. New York: Academic Press.

Chakkalakal DA, Wilson RF Jr, Connolly JF (1988) Epidermal and endosteal sources of endogenous electricity in injured canine limbs. IEEE Trans Biomed Eng 35:19-30.

Bachmann $\mathrm{T}$ (1984) The process of perceptual retouch: nonspecific afferent activation dynamics in explaining visual masking. Percept Psychophys 35:69-84.

Cui RQ, Deecke L (1999) High resolution DC-EEG analysis of the Bereitschaftspotential and post movement onset potentials accompanying uni- or bilateral voluntary finger movements. Brain Topogr 11:233-49.

Caspers H, Speckmann EJ, Lehmenkühler A (1980) Electrogenesis of cortical DC potentials. Prog Brain Res 54:3-15.

Caspers H, Speckmann E-J, Lehmenkühler A (1984) Electrogenesis of slow potentials of the brain In: Self-regulation of the brain and behavior (Elbert T, Rockstroh B, Birbaumer N, eds.), pp 26-41. Berlin: Springer.

Caspers H (1993) DC potentials of the brain. In: Slow potential changes in the brain (Haschke W, Speckmann EJ, Roitbak A, eds.), pp 9-21. Boston: Birkhäuser.

Dreier JP, Major S, Manning A, Woitzik J, Drenckhahn C, Steinbrink J, Tolias C, OliveiraFerreira AI, Fabricius M, Hartings JA, Vajkoczy P, Lauritzen M, Dirnagl U, Bohner G, Strong AJ; COSBID study group (2009) Cortical spreading ischaemia is a novel process involved in ischaemic damage in patients with aneurysmal subarachnoid haemorrhage. Brain 132:1866-81.

Dreier JP, Major S, Pannek HW, Woitzik J, Scheel M, Wiesenthal D, Martus P, Winkler MK, Hartings JA, Fabricius M, Speckmann EJ, Gorji A; COSBID study group (2012) Spreading convulsions, spreading depolarization and epileptogenesis in human cerebral cortex. Brain 135:259-75. 
Drenckhahn C, Winkler MK, Major S, Scheel M, Kang EJ, Pinczolits A, Grozea C, Hartings JA, Woitzik J, Dreier JP; COSBID study group (2012) Correlates of spreading depolarization in human scalp electroencephalography. Brain 135:853-68.

Eickhoff M, Kovac S, Shahabi P, Ghadiri MK, Dreier JP, Stummer W, Speckmann EJ, Pape HC, Gorji A (2014) Spreading depression triggers ictaform activity in partially disinhibited neuronal tissues. Exp Neurol 253:1-15.

Geddes LA (1997) Historical evolution of circuit models for the electrode-electrolyte interface. Ann Biomed Eng 25: 1-14.

Gollwitzer S, Groemer T, Rampp S, Hagge M, Olmes D, Huttner HB, Schwab S, Madžar D, Hopfengaertner R, Hamer HM (2015) Early prediction of delayed cerebral ischemia in subarachnoid hemorrhage based on quantitative EEG: A prospective study in adults. Clin Neurophysiol 126:1514-23.

Gorji A (2001) Spreading depression: a review of the clinical relevance. Brain Res Brain Res Rev 38:33-60.

Gorji A, Scheller D, Straub H, Tegtmeier F, Köhling R, Höhling JM, Tuxhorn I, Ebner A, Wolf P, Werner Panneck H, Oppel F, Speckmann EJ (2001) Spreading depression in human neocortical slices. Brain Res 906:74-83.

Hadjikhani N, Sanchez Del Rio M, Wu O, Schwartz D, Bakker D, Fischl B, Kwong KK, Cutrer FM, Rosen BR, Tootell RB, Sorensen AG, Moskowitz MA (2001) Mechanisms of migraine aura revealed by functional MRI in human visual cortex. Proc Natl Acad Sci U S A 98:4687-92.

Haider M, Groll-Knapp E, Ganglberger JA (1981) Event-related slow (DC) potentials in the human brain. Rev Physiol Biochem Pharmacol 88:125-97.

Hallschmid M, Mölle M, Wagner U, Fehm HL, Born J (2001) Drinking related direct current positive potential shift in the human EEG depends on thirst. Neurosci Lett 311:173-6.

Hartings JA, Bullock MR, Okonkwo DO, Murray LS, Murray GD, Fabricius M, Maas AI, Woitzik J, Sakowitz O, Mathern B, Roozenbeek B, Lingsma H, Dreier JP, Puccio AM, Shutter LA, Pahl C, Strong AJ; Co-Operative Study on Brain Injury Depolarisations (2011) Spreading depolarisations and outcome after traumatic brain injury: a prospective observational study. Lancet Neurol 10:1058-64.

Hartings JA, Wilson JA, Hinzman JM, Pollandt S, Dreier JP, DiNapoli V, Ficker DM, Shutter LA, Andaluz N (2014) Spreading depression in continuous electroencephalography of brain trauma. Ann Neurol 76:681-94.

Ikeda A, Terada K, Mikuni N, Burgess RC, Comair Y, Taki W, Hamano T, Kimura J, Lüders HO, Shibasaki H (1996) Subdural recording of ictal DC shifts in neocortical seizures in humans. Epilepsia 37:662-74. 
Kamp A, Pfurtscheller G, Edlinger G, Lopes da Silva F (2005) Polygraphy. In: Electroencephalography, Basic principles, Clinical applications and related fields (Niedermeyer E, Lopes da Silva F, eds.), pp 891-898. Philadelphia: Lippincott Williams\&Wilki.

Kappenman ES, Luck SJ (2010) The effects of electrode impedance on data quality and statistical significance in ERP recordings. Psychophysiology 47:888-904.

Khaleghi Ghadiri M, Tutam Y, Wassmann H, Speckmann EJ, Gorji A (2009) Periodic fasting alters neuronal excitability in rat neocortical and hippocampal tissues. Neurobiol Dis 36:38492.

Knoll O, Speckmann EJ, Caspers H (1974) Ein Verfahren zur Korrelierung verschiedener bioelektrischer Vorgänge mit definierten Potentialmustern im EEG. Z. EEG EMG, 5:199-205.

Knoll O, Speckmann EJ (1973) Pattern related event distribution of untriggered signals. Decus Europe Proceedings: Ninth Seminar, pp 213-217.

Leao AA (1947) Further observations on the spreading depression of activity in the cerebral cortex. J Neurophysiol 10:409-414.

Lee S, Kruse J (2016) Biopotential Electrode Sensors in ECG/EEG/EMG Systems. Analog Devices, Inc., http://www.analog.com/media/en/technical-documentation/white-papers/ECGEEG-EMG_FINAL.pdf, Retrieved 29 January 2016.

Lehmankühler A, Richter F (1993) Spreading depression in upper and lower depths of the rat cerebral cortex and its possible implications on the type of human migraine. In: Migraine: Basic Mechanisms and Treatment (Lehmankühler A, Grotemeyer K-H, Tegtmeier F, eds.), pp 267-278. Munich, Vienna, Baltimore: Urban \& Schwarzberg.

Lehmenkühler A, Richter F, Pöppelmann T (1999) Hypoxia- and hypercapnia-induced DC potential shifts in rat at the scalp and the skull are opposite in polarity to those at the cerebral cortex. Neurosci Lett 270:67-70.

Macy A (2016) The Handbook of Human Physiological Recording. Chapter 4: electrodes. http://www.alanmacy.com/HPR-Chapter4.html. Retrieved 29 January 2016.

Mansfeld F, Lin S, Chen YC, Shih H (1988) Minimization of High-Frequency Phase Shifts in Impedance Measurements. JES 135:906.

Mesgari M, Ghaffarian N, Khaleghi Ghadiri M, Sadeghian H, Speckmann EJ, Stummer W, Gorji A (2015) Altered inhibition in the hippocampal neural networks after spreading depression. Neuroscience 304:190-7. 
Niedermeyer E, da Silva FL (2004) Electroencephalography: Basic Principles, Clinical Applications, and Related Fields. Philadelphia: Lippincot Williams \& Wilkins.

Rodin E, Constantino T, van Orman C, House P (2008) EEG infraslow activity in absence and partial seizures. Clin EEG Neurosci 39:12-9.

Saadi H, Attari M (2013) Electrode-gel-skin interface characterization and modeling for surface biopotential recording: Impedance measurements and noise. 2nd International Conference on Advances in Biomedical Engineering, pp. 49-52.

Schmidinger A, Greiner C, Reinker S, Köhling R, Lücke A, Straub H, Speckmann E, Moskopp D, Wassmann H, Lahl R, Pannek H, Oppel F (1998) Flat and steep terminal negativity in the DC-potential after deprivation of oxygen and glucose in human neocortical slices. Brain Res 794:28-34.

Seghatoleslam M, Ghadiri MK, Ghaffarian N, Speckmann EJ, Gorji A (2014) Cortical spreading depression modulates the caudate nucleus activity. Neuroscience 267:83-90.

Staschen CM, Lehmenkühler A, Zidek W, Caspers H (1987) Relations between the cortical DC potentials and the $\mathrm{K}+$ concentration of the blood and cerebral cortex extracellular space in reversible asphyxia. EEG EMG Z Elektroenzephalogr Elektromyogr Verwandte Geb 18:53-7.

Somjen GG (2001) Mechanisms of spreading depression and hypoxic spreading depressionlike depolarization. Physiol Rev 81:1065-1096.

Speckmann E-J, Elger CE, Gorji A (2011) Neurophysiological Basis of EEG and DC Potential, In: Niedermeyer's Electroencephalography (Schomer DL; Lopes da Silva F, eds.), pp. 17-31. Philadelphia: Lippincott Williams \& Wilkins.

Tallgren P, Vanhatalo S, Kaila K, Voipio J (2005) Evaluation of commercially available electrodes and gels for recording of slow EEG potentials. Clin Neurophysiol 116:799-806.

Trimmel M, Strässler F, Knerer K (2001) Brain DC potential changes of computerized tasks and paper/pencil tasks. Int J Psychophysiol 40:187-94.

Tomita-Gotoh S, Hayashida Y (1996) Scalp-recorded direct current potential shifts induced by hypocapnia and hypercapnia in humans. Electroencephalogr Clin Neurophysiol 99:90-7.

Van Harreveld A, Stamm JS (1954) Relation between asphyxial damage to the cortex and the spreading depression. Am J Physiol 178:117-22.

Vanhatalo S, Voipio J, Kaila K (2005) Full-band EEG (FbEEG): an emerging standard in electroencephalography. Clin Neurophysiol 116:1-8. 
Walden J, Speckmann EJ (1988) Suppression of recurrent generalized tonic-clonic seizure discharges by intraventricular perfusion of a calcium antagonist. Electroencephalogr Clin Neurophysiol 69:353-62.

Wong D, Clifton DA, Tarassenko L (2009) An Introduction To The Bispectrum for EEG Analysis. Postgraduate Conference in Biomedical Engineering \& Medical Physics, pp 61-62. 


\section{Legends of figures}

Fig. 1. Analysis of a trace of DC potential shift recorded by EEG in rat. The solid green line is the mean $\mu$ of the first 5 seconds of the signal and the dashed lines are the mean and standard deviation $(\mu+\sigma$ and $\mu-\sigma)$ of the EEG signal amplitude. The beginning of DC signal is the moment that the EEG signal amplitude crosses one of dashed lines and starts to assume the shape of the standard SD morphology. The end of the DC signal is marked when the negative low amplitude component (the third component) of DC signal returns between the dashed lines.

Fig. 2. Recording of slow propagation of DC potential deflections by ECoG, scalp (EEG) 1, and scalp (EEG) 2 electrodes after induction of spreading depression (SD) in rat brain. SD was induced by $\mathrm{KCl}$ injection. Inset on the top shows the position of injection and recording electrodes (A). Spectrograms corresponding to the ECoG and scalp (EEG) 1 as well as scalp (EEG) 2 signals presented in figure 3. Power spectrum is adjusted to the frequency of up to 3Hz. The color is mapped from red (presents the highest energy) to blue (presents the lowest energy). The spectrogram of signals recorded during initiation and propagation of SD revealed spreading high-energy signals in a frequency between 0.05 to $0.1 \mathrm{~Hz}$ recorded by ECoG and scalp (EEG) electrodes during propagation of SD (B).

Fig. 3. Bi-spectrum analysis of DC potential recorded by ECoG electrode before, during and after induction of DC potential fluctuation. Different time points for analysis are shown on the DC potential shift recorded by ECoG electrodes. The green and light blue colors and red contours around the main central red ellipse represent the emergence of higher frequency waves in ECoG (A-B). Disappearance of higher frequencies of bioelectrical activities of the brain at the beginning of negative DC potentials indicates depression of the brain activity after SD (B-E). After SD, red ellipse started to being more confined and the small red contours 
around the main counter are completely disappeared. This finding points to the reversible depression of brain waves of negative DC potentials on brain bioelectrical activities (F-K).

Fig. 4. Bi-spectrum analysis of DC potential recorded by scalp (EEG) electrode before, during and after DC potential deflection. Different time points for analysis are shown on the DC potential shift recorded by scalp (EEG) electrodes. The green and light blue colors and red contours around the main central red ellipse indicate the emergence of higher frequency waves in EEG (A-B). Disappearance of higher frequencies of bioelectrical activities of the brain at the beginning of negative DC potentials indicates depression of the brain activity after SD (B-E). After SD, red ellipse started to being more confined and the small red contours around the main counter are completely disappeared. This result points to reversible depressive effect of negative DC wave on neuronal activities (F-K).

Fig. 5. Correlation and the multivariable Gaussian fit analysis of the amplitude and duration of DC potential shifts recorded by ECoG, scalp (EEG) 1, and scalp (EEG) 2 electrodes. Correlation of the amplitude and duration of DC waves recorded by ECoG and scalp 1 electrodes (A). Correlation of the amplitude and duration of DC potentials recorded by scalp 1 and scalp 2 electrodes. The ellipses show the multivariable Gaussian fit analysis. The blue ellipses fitted to the farthest data points, the red ellipse fitted to the nearest data points, and green or red ellipses are fitted to the middle points $(\mathbf{B})$.

Fig. 6. To evaluate the homogenous pattern of negative DC shifts recorded by ECoG and scalp (EEG) 1 electrodes after induction of spreading depression, bioelectrical potentials were synchronized, normalized and then compared for alteration of signal morphology. Blue and red traces are normalized potentials recorded by ECoG and scalp 1, respectively. Black trace is the subtraction of synchronized and normalized DC potentials of ECoG and scalp1 recordings. 
Fig. 7. To study the homogenous pattern of negative DC waves recorded by scalp (EEG) 1 and scalp (EEG) 2 electrodes after induction of spreading depression, DC potentials were synchronized, normalized and then compared for changes of signal morphology. Red and blue traces are normalized potentials recorded by scalp 1 and scalp 2, respectively. Black trace is the subtraction of synchronized and normalized DC waves recorded by scalp 1 and scalp 2 . 


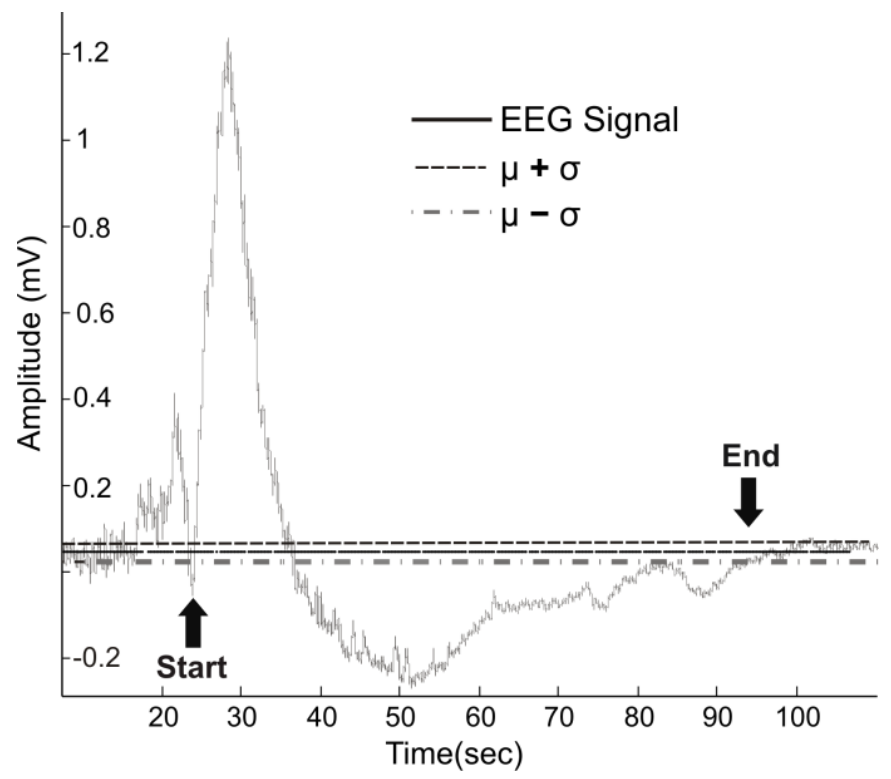


A
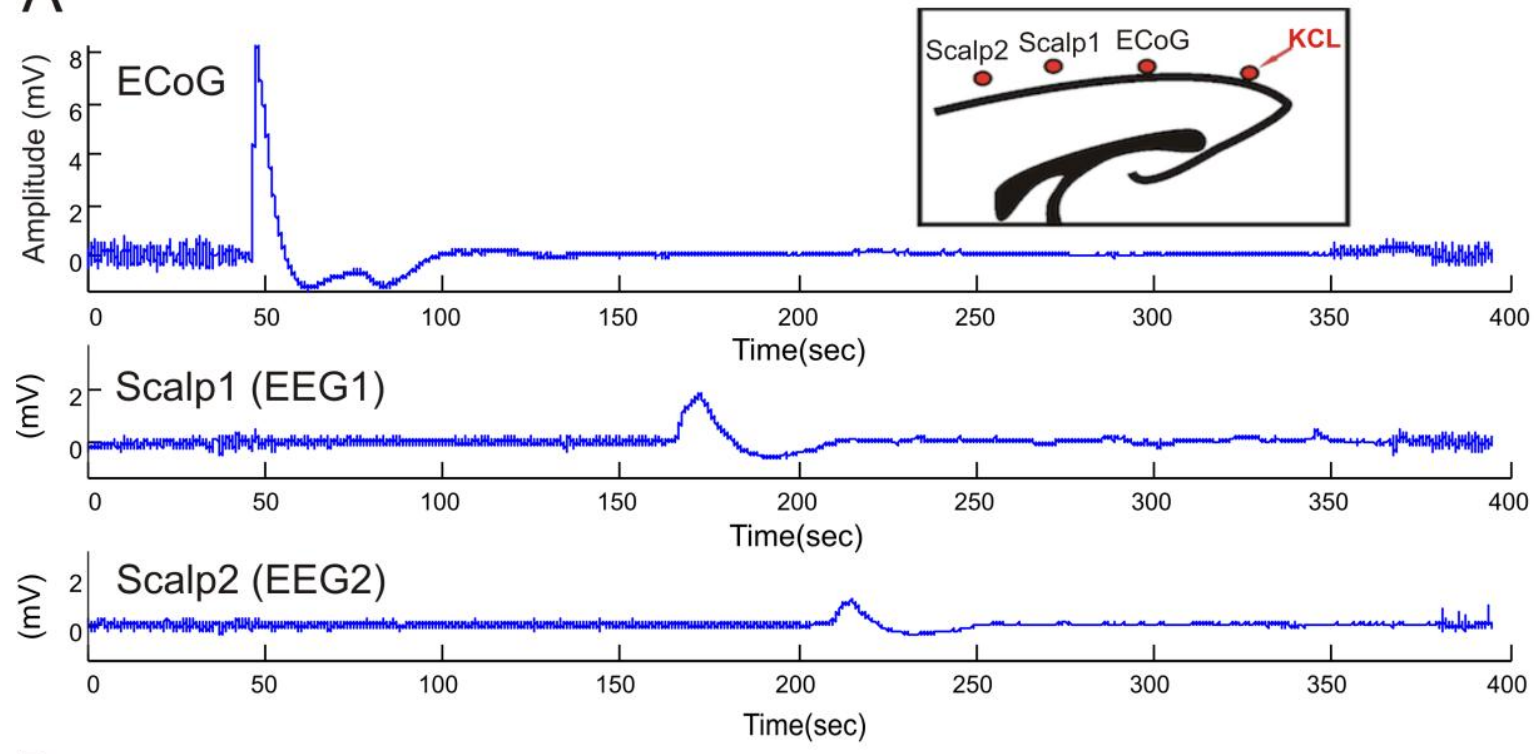

B

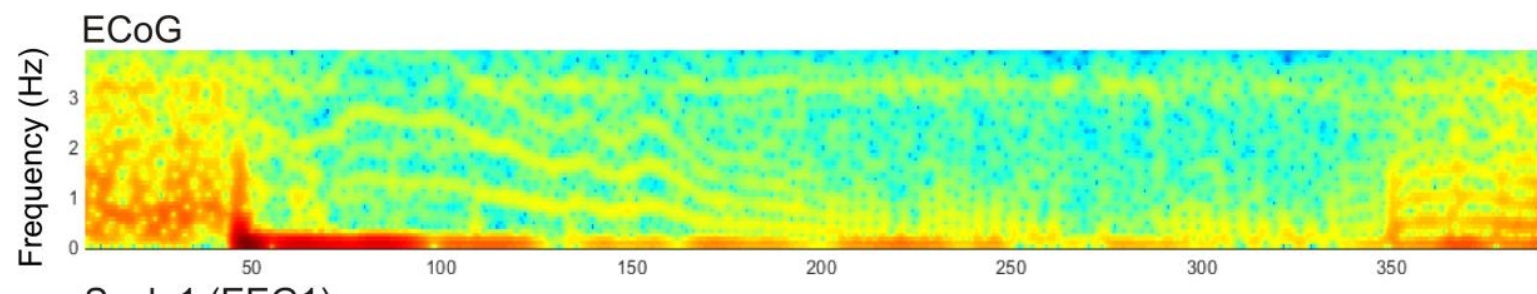

Scalp1 (EEG1)

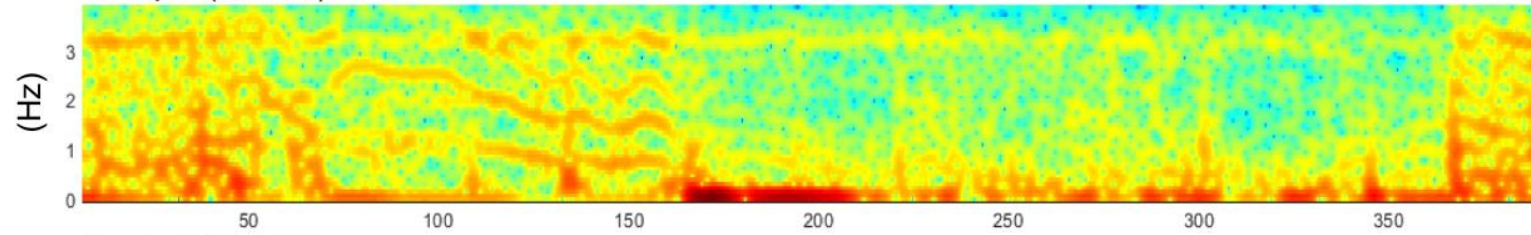

Scalp2 (EEG2)

Nㅗ․

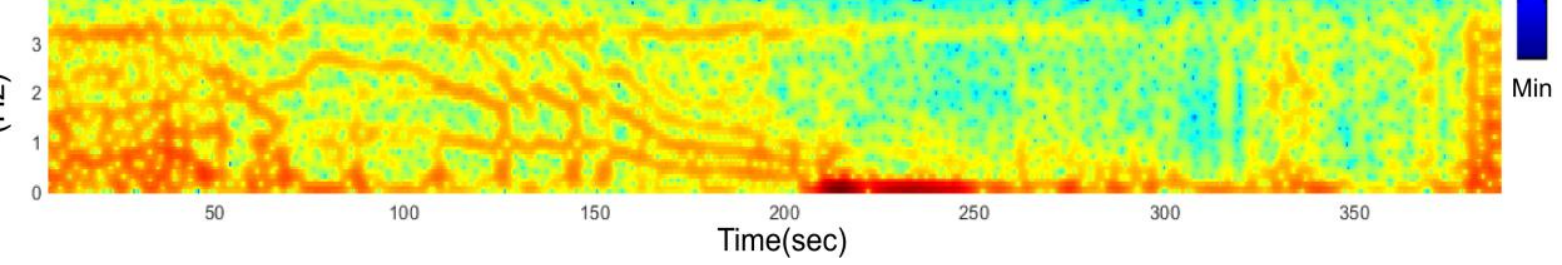



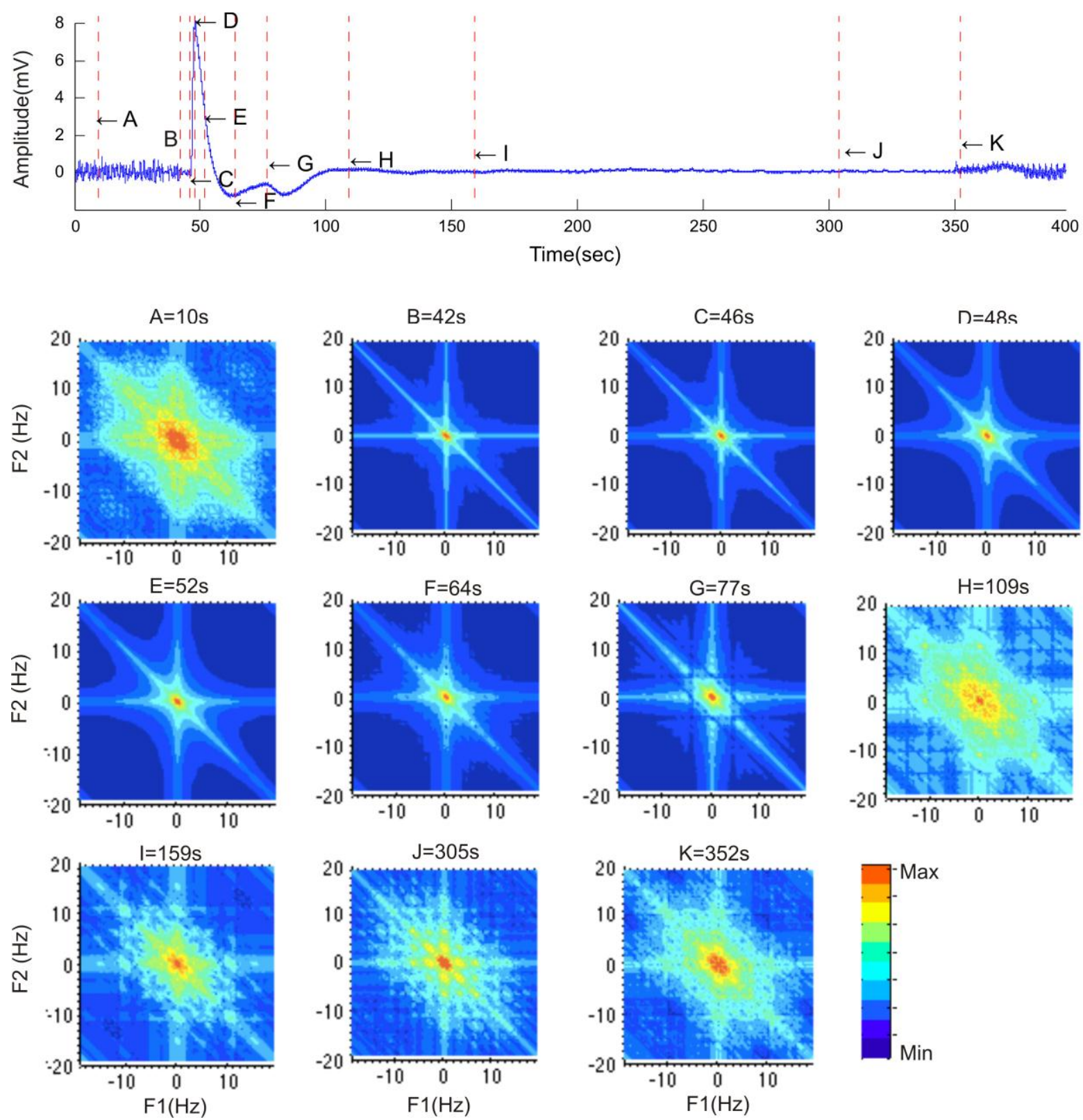

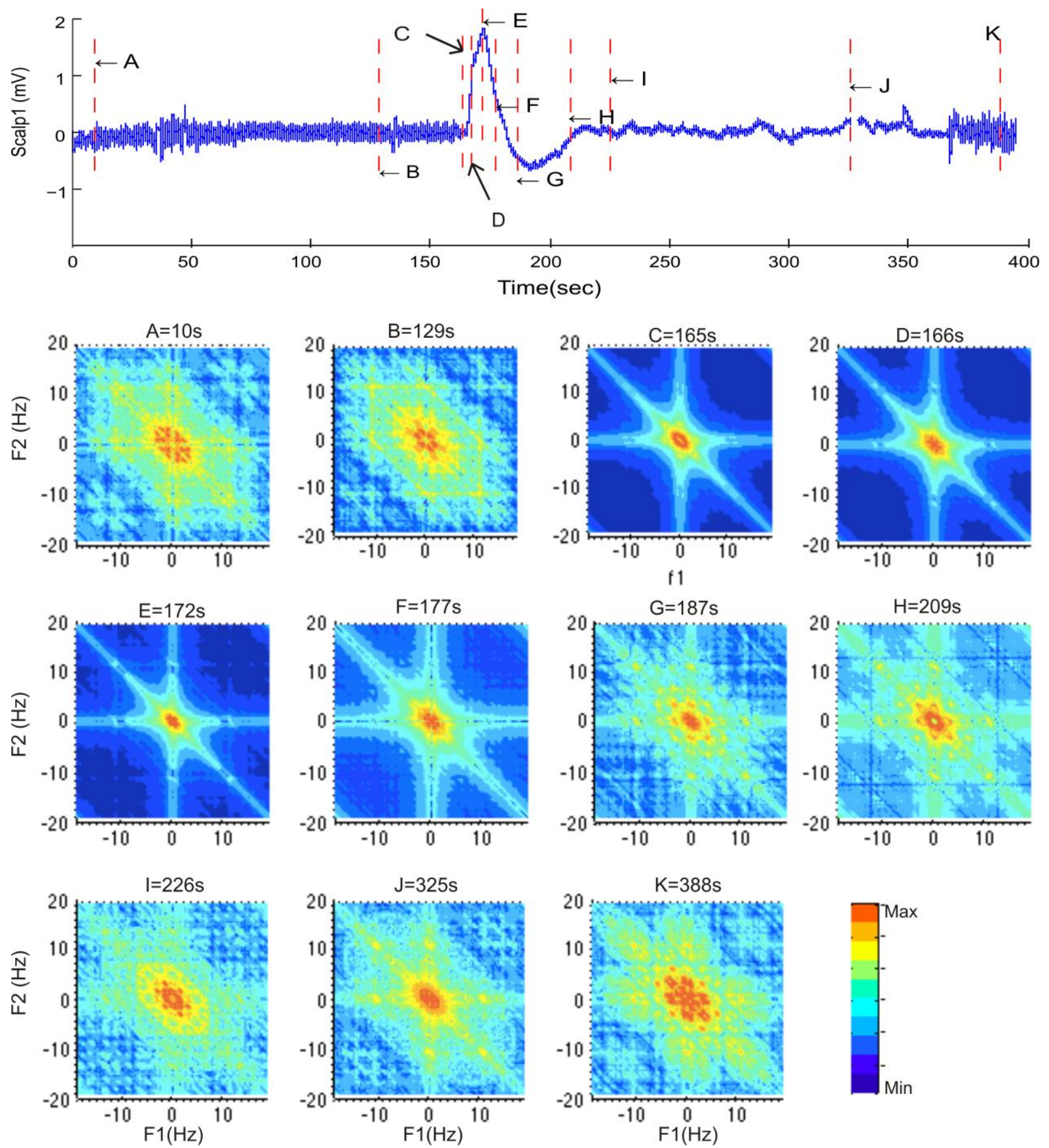

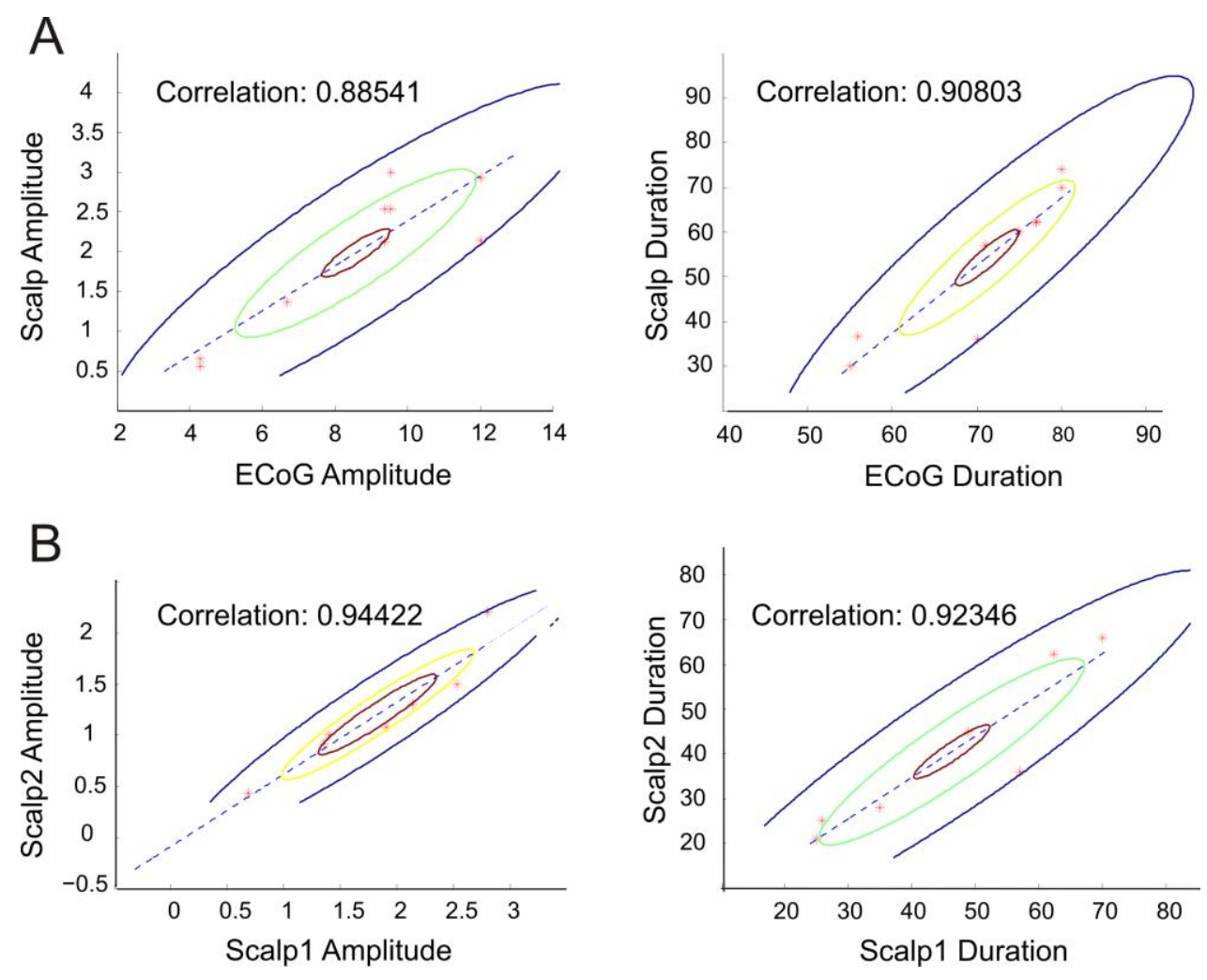


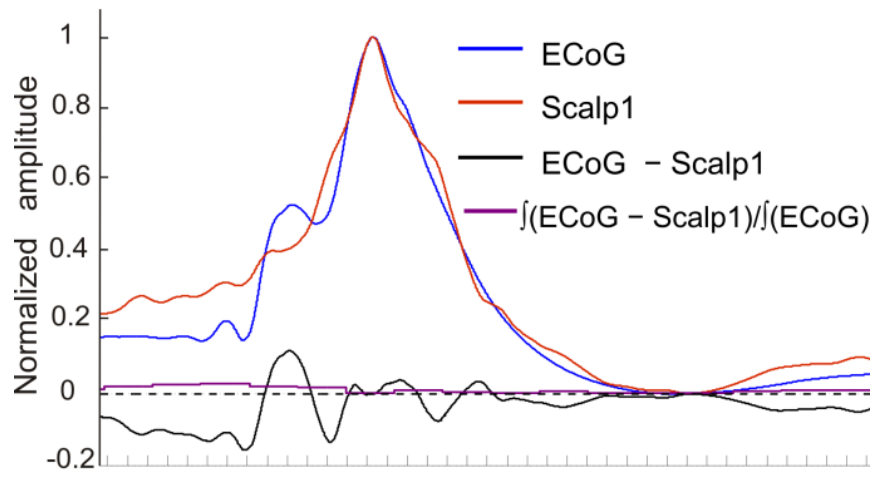




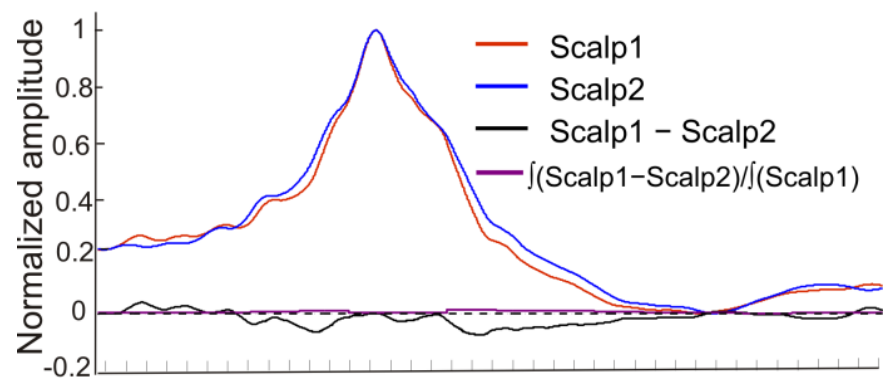

